\title{
Manejo en el paciente hospitalizado por COVID-19
} Inpatient management of diabetes and COVID-19

\author{
Fériz-Bonelo $K^{1}$
}

${ }^{1}$ Médica Internista - Endocrinóloga, Fundación Valle del Lili. Docente, posgrado de Medicina Interna, ICESI-CES. Coordinadora, Comité Diabetes, Asociación Colombiana de Endocrinología. Miembro de número, Asociación Colombiana de Endocrinología.

Autor de correspondencia: Karen Fériz Bonelo

Correo electrónico: kaferiz@hotmail.com

\section{Resumen}

La diabetes es un factor de riesgo claramente establecido para la COVID-19 y para sus formas graves, lo que aumenta, entonces, el riesgo de hospitalización por infección por SARSCoV-2 en los pacientes que la padecen. En el mundo entero existe poca evidencia con respecto a la mejor estrategia de manejo del paciente con diabetes que es hospitalizado por COVID-19. En términos generales, la insulina, ya sea subcutánea o intravenosa, constituye el tratamiento de elección. El uso de antidiabéticos orales no está estudiado y no se recomienda como primera línea. Las metas de control glucémico son las mismas que para la población general. La necesidad de control glucométrico frecuente, principalmente en aquellos con infusión intravenosa (IV) de insulina, constituye el reto más importante en el manejo de estos pacientes, pues aumenta la necesidad de personal disponible (el cual puede ser escaso en época de crisis) y la exposición del personal de salud a la infección.

Palabras clave: diabetes, hospitalizados, manejo, COVID-19, SARS-CoV-2.

\begin{abstract}
Diabetes is a clearly established risk factor for COVID-19 and for severe forms of it, increasing the risk of hospitalization for SARS-CoV-2 infection in patients with it. There isn't enough evidence worldwide regarding the best management strategy for a patient with diabetes who is hospitalized for COVID-19. Insulin, either subcutaneous or intravenous, is the treatment of choice; the use of oral antidiabetics is not studied and is not recommended as the first line. Glycemic control goals are the same as for the general population. The need for frequent glucometric control, mainly in those with IV insulin infusion,
\end{abstract}

constitutes the most important challenge in the management of these patients, since it increases the need for available personnel (which may be scarce in times of crisis) and the exposure of health to infection.

Keywords: Diabetes, Inpatient, Management, COVID-19, SARS-CoV-2.

\section{Introducción}

Hoy día la diabetes mellitus (DM) constituye un factor de riesgo claramente establecido de gravedad de la infección por SARS-CoV-2 y mortalidad asociada, como fue previamente descrito ${ }^{(1,2)}$. Existe, además, evidencia que sugiere que la hiperglucemia no controlada (en población con o sin diabetes previamente conocida) se asocia a mayor mortalidad y estancia hospitalaria prolongada ${ }^{(3)}$.

Los pacientes con DM o hiperglucemia son significativamente de mayor edad, de género masculino y tienen mayor compromiso de su función renal cuando son comparados con los no diabéticos que son hospitalizados ${ }^{(3)}$.

La hiperglucemia ha demostrado ejercer múltiples efectos a nivel del tracto respiratorio, que podrían empeorar el pronóstico de los pacientes diabéticos con infecciones virales respiratorias ${ }^{(4)}$, como se discutió previamente. Por otro lado, la infección per se puede generar una respuesta inflamatoria sistémica grave con liberación de catecolaminas y cortisol y puede ocasionar daño directo a las células betapancreáticas, lo cual empeora la hiperglucemia y aumenta la variabilidad glucémica ${ }^{(5)}$.

Teniendo en cuenta el gran número de pacientes críticamente enfermos con COVID-19 y diabetes o hiperglucemia y los desenlaces adversos asociados a esta condición, se hace necesario un manejo oportuno, eficaz y estandarizado de la glucemia en este escenario.

\section{Control glucémico intrahospitalario}

Lograr un adecuado control glucémico en el paciente con DM y COVID-19 no ha sido fácil, una vez que existe poca disponibilidad de endocrinólogos en los sitios de aislamiento destinados a la atención de estos pacientes. Hay dificultades con el 
mantenimiento de una dieta adecuada para diabetes en el contexto de la hospitalización y la ansiedad de los pacientes ante su diagnóstico, que tiene un impacto negativo sobre la glucemia y el efecto per se del virus sobre las células betapancreáticas.

En términos generales, la elección del tratamiento debe estar centrada en las características individuales de cada paciente, como la edad, las comorbilidades, el riesgo de hipoglucemia y la gravedad de la infección por SARS-CoV-2. La evidencia de uso de antidiabéticos orales en el paciente hospitalizado es escasa. En términos generales, se acepta que, en el hospital, sea suspendida la metformina por el riesgo de insuficiencia respiratoria e hipoxemia asociada a la infección, con el consecuente riesgo de acidosis; y las sulfonilúreas, por el riesgo de hipoglucemia. Los agonistas del receptor GLP-1 y los inhibidores del glucotransportador de sodio-glucosa (iSGLT-2) podrían, en teoría, asociarse a peores desenlaces, pues inducen una sobreexpresión de la enzima convertidora de la angiotensina 2 (ECA-2), aunque no existen datos en humanos y, por otro lado, dado su mecanismo de acción, podrían ejercer un efecto antinflamatorio que sería benéfico, así que no hay, hasta la fecha, una recomendación puntual con los GLP-1; sin embargo, con los iSGLT-2 existe riesgo de cetoacidosis diabética si el paciente tiene depleción del volumen asociada a la infección viral, por lo cual se recomienda sean suspendidos. Finalmente, existe un potencial papel protector de los inhibidores de la enzima dipeptidil peptidasa-4 (DPP-4) asociado a su efecto antiinflamatorio, que podría reducir los desenlaces pulmonares adversos, aunque esto no está demostrado ${ }^{(6)}$.

En pacientes hospitalizados, pero no críticamente enfermos, la terapia ideal es insulina subcutánea en esquema de dosis fijas (basal más corrección o basal-bolo) según el estado y las necesidades del paciente.

Los pacientes críticamente enfermos con glucemia $>180$ $\mathrm{mg} / \mathrm{dL}$ deben recibir infusión intravenosa (IV) de insulina (con un aumento importante en la necesidad de dosis de insulina en DM2 por el incremento considerable de la insulinorresistencia en el marco de la infección); sin embargo, el ajuste de la dosis según la glucemia del paciente representa uno de los principales retos en su manejo, ya que para este se requiere monitorizar los niveles de glucosa cada hora o cada dos horas (hasta que la glucosa del paciente esté estable), lo cual implica la necesidad de personal entrenado disponible (que puede escasear en tiempos de crisis) y un aumento de la exposición del personal de salud a la infección. En casos en los que el sistema de salud se desborda y los recursos intrahospitalarios se agotan, puede verse comprometida la disponibilidad de bombas de infusión para la administración de goteos de insulina. Para tratar de vencer estas barreras, se han propuesto alternativas diferentes de manejo de la hiperglucemia en la unidad de cuidados intensivos (UCI), como el uso de análogos de acción corta vía subcutánea, cada 1 o 2 horas (usados con éxito en el manejo de la cetoacidosis diabé- tica leve o moderada), aunque su seguridad en el entorno de la UCI no ha sido evaluada ${ }^{(7)}$. Algunos expertos sugieren que el uso de insulina basal con correcciones con insulina rápida cada 4 horas podría ser otra opción de manejo para reducir la frecuencia de glucometrías; sin embargo, su eficacia y seguridad no han sido evaluadas ${ }^{(8)}$.

La Figura 1 presenta un abordaje inicial del paciente en el hospital ${ }^{(9)}$.

La glucosa debe ser monitorizada de manera regular durante toda la hospitalización de los pacientes con infección por COVID-19 y DM, con el objetivo de instaurar un manejo oportuno, si no se cumplen las metas, y de identificar tempranamente un empeoramiento de la glucemia en relación con el tratamiento instaurado (uso de glucocorticoides, por ejemplo).

La meta de glucemia es la misma que en población que es hospitalizada por otra causa: $<140 \mathrm{mg} / \mathrm{dL}$ antes de las comidas y $<180 \mathrm{mg} / \mathrm{dL}$ después de las comidas.

Debe tenerse en cuenta que el uso de acetaminofén puede inducir errores en las lecturas de las glucometrías o en los datos de la monitorización continua de la glucosa (depende del tipo de glucómetro o monitorización utilizados).

La frecuencia en la realización de las glucometrías representa otro reto importante en el cuidado de estos pacientes, pues exige, sobre todo en aquellos con insulina IV, contacto frecuente con el paciente. Para minimizar los riesgos para el personal a cargo, se sugiere que, si el paciente no está críticamente enfermo, se mida él mismo la glucosa y la comunique vía telefónica, para hacer los ajustes necesarios. El uso de monitorización continua de la glucosa puede ser otra opción, en especial si se usa tecnología que permita un acceso remoto a los datos; sin embargo, la falta de evidencia de su eficacia en el contexto hospitalario, el costo y la falta de disponibilidad constituyen una limitante para su uso en nuestro medio.

\section{Hiperglucemia aguda en pacientes sin diabetes mellitus}

Históricamente, la hiperglucemia de estrés (glucosa $>180$ $\mathrm{mg} / \mathrm{dL}$ en pacientes sin historia previa de diabetes, con $\mathrm{HbA1c}$ $<6,5 \%$ y que cursa con una enfermedad aguda) ha sido asociada a peores desenlaces clínicos, incluida una mayor mortalidad, al compararse con población normoglucémica e incluso con población con DM previamente diagnosticada. De la misma manera, durante la epidemia del síndrome respiratorio agudo grave (SARS) en el 2003, esta se identificó como una complicación de la infección y se asoció a mayor riesgo de falla respiratoria y muerte ${ }^{(10)}$. Datos actuales que estudian la relación entre la COVID-19 y la hiperglucemia también sugieren la misma asociación. En un estudio descriptivo en Estados Unidos, durante un análisis de subgrupos de 184 pacientes, se reportó que la mortalidad fue significativamente mayor en aquellos con hiperglucemia aguda sin DM que en los pacientes 
Figura 1. Abordaje inicial del paciente en el servicio de urgencias. Adaptada de la referencia 9. IV: intravenoso; iSGLT-2: inhibidores del glucotransportador de sodio-glucosa; SC: subcutáneo.

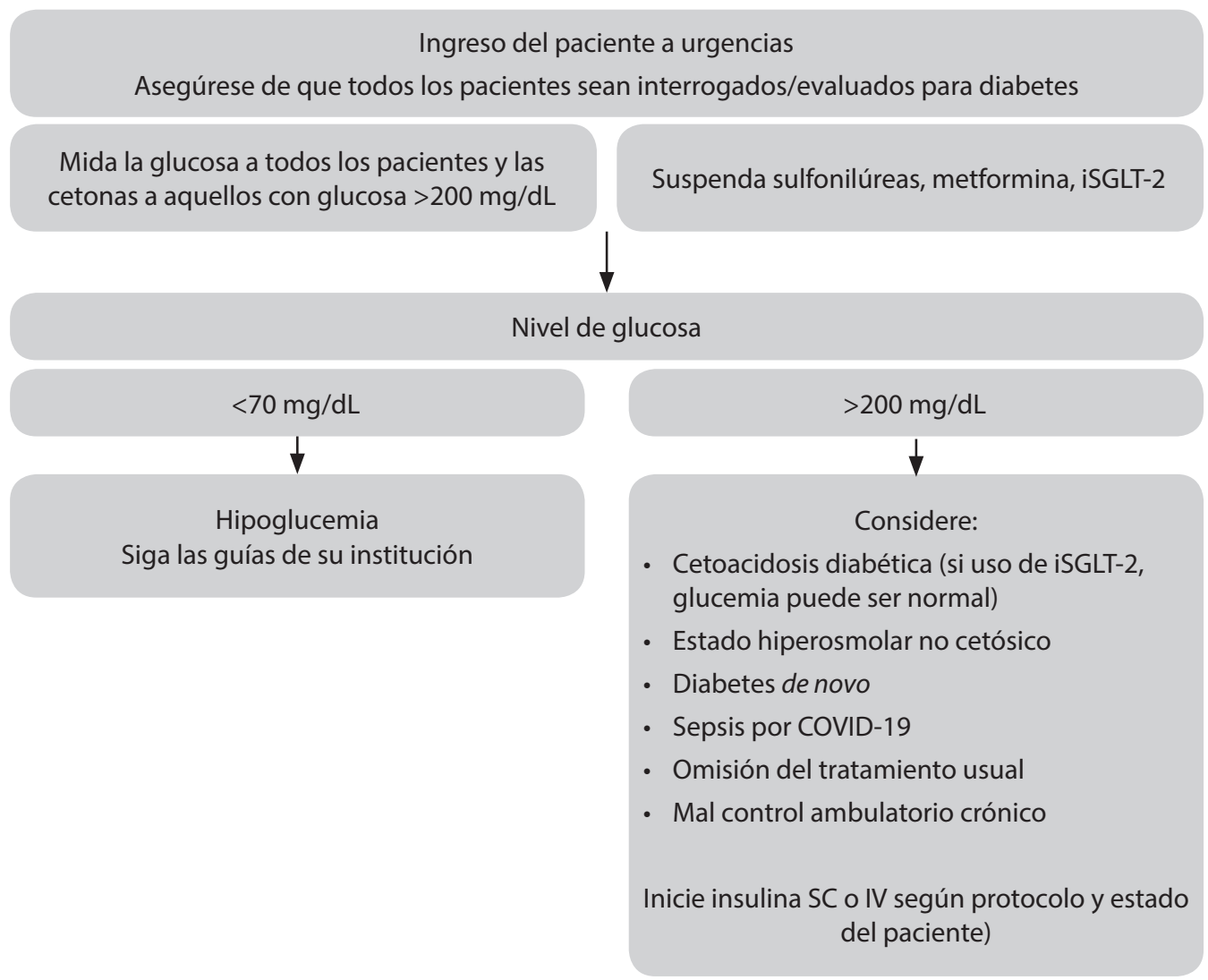

con DM conocida (41,7\% vs. 14,8\%; $p<0,001)$; igualmente, la estancia hospitalaria fue significativamente mayor en el grupo de hiperglucemia aguda vs. el grupo de DM (6,2 vs. 5 días; $p$ $<0,001)^{(3)}$.

Ante los desenlaces adversos asociados, la hiperglucemia aguda en pacientes sin DM podría interpretarse como un indicador de daño de la célula betapancreática y considerarse como un factor de riesgo para peor pronóstico. Hasta hoy, no existe evidencia que demuestre que el manejo de la hiperglucemia aguda en el contexto de la COVID-19 mejore los desenlaces clínicos de los que la presentan; sin embargo, la recomendación aceptada es que esta sea manejada de la misma manera que los pacientes hospitalizados por otra causa, con una meta de $<180 \mathrm{mg} / \mathrm{dL}^{(11)}$.

\section{Hipoglucemia}

La hipoglucemia es una complicación del manejo de la diabetes, que ocurre más frecuentemente en aquellos que reciben terapia con insulina. La presencia de hipoglucemia grave en el paciente hospitalizado se asocia a una mayor mortalidad (12). En el contexto de la infección por COVID-19, una de las estrategias farmacológicas planteadas es la administración de cloroquina o hidroxicloroquina, fármacos cuyo uso ha estado asociado a hipoglucemia ${ }^{(13)}$, razón por la cual debe hacerse seguimiento estrecho con glucometrías y ajuste oportuno de la dosis de ADOS o insulina a quienes reciban este fármaco. Debe tenerse atención especial al paciente pronado, ya que la alimentación puede interrumpirse de manera accidental en esta posición, lo que generará mayor riesgo de hipoglucemia.

\section{Conflicto de interés}

El autor certifica que no tiene conflictos de interés para la elaboración de este manuscrito.

\section{Financiación}

El autor declara que no recibió recursos para la elaboración de este manuscrito. 


\section{Referencias}

1. Wu Z, McGoogan JM. Characteristics of and important lessons from the coronavirus disease 2019 (COVID-19) outbreak in China: Summary of a report of 72314 cases from the Chinese Center for Disease Control and Prevention. JAMA. 2020. doi: https://doi.org/10.1001/jama.2020.2648 February 24.

2. Guan WJ, Ni ZY, Hu Y, Liang WH, OU CQ, He JX, et al. Clinical Characteristics of Coronavirus Disease 2019 in China. New Engl J Med. 2020;382(18):170820. doi: $10.1056 /$ NEJMoa2002032.

3. Bode B, Garret V, Messler J, McFarland R, Crowe J, Booth R, et al. Glycemic Characteristics and Clinical Outcomes of COVID-19 Patients Hospitalized in the United States. J Diabetes Sci Technol. 2020;193229682094469. doi: $10.1177 / 1932296820924469$.

4. Hill M, Mantzoros C, Sowers JR. Commentary: COVID-19 in patients with diabetes. Metabolism. 2020;107:154217. doi: 10.1016/j.metabol.2020.154217.

5. Wang A, Zhao W, Xu Z, Gu J. Timely blood glucose management for the outbreak of 2019 novel coronavirus disease (COVID-19) is urgently needed. Diabetes Res Clin Pract. 2020;162:108118. doi: 10.1016/j.diabres.2020.108118.

6. Pal R, Bhadada SK. Should anti-diabetic medications be reconsidered amid COVID-19 pandemic? Diabetes Res Clin Pract. 2020;163:108146. doi: 10.1016/j.diabres.2020.108146
7. Umpierrez G, Cuervo R, Karabell A, Latif K, Freire AX, Kitabchi AE, et al. Treatment of Diabetic Ketoacidosis With Subcutaneous Insulin Aspart. Diabetes Care. 2004;27(8):1873-8. doi: 10.2337/diacare.27.8.1873.

8. Hirsch I, Umpierrez G. Inpatient care and COVID-19 [Internet]. Disponible en: https://professional.diabetes.org/content-page/covid-19.

9. National Inpatient Diabetes COVID-19 Response Team. Concise Advice on Inpatient Diabetes (COVID:Diabetes): Front Door Guidance [Internet]. Disponible en: https://bit.ly/3dg5FMT.

10. Yang JK, Feng Y, Yuan MY, Yuan SY, Fu HJ, Wu BY, et al. Plasma glucose levels and diabetes are independent predictors for mortality and morbidity in patients with SARS. Diabet Med. 2006;23(6):623-8. doi: 10.1111/j.14645491.2006.01861.x.

11. American Diabetes Association. 15. Diabetes Care in the Hospital: Standards of Medical Care in Diabetes-2020. Diabetes Care. 2020;43(Suppl 1):S193 S202. doi: $10.2337 / \mathrm{dc} 20-\mathrm{S} 015$

12. Akirov A, Grossman A, Shochat T, Shimon I. Mortality among hospitalized patients with hypoglycemia: insulin related and noninsulin related. J Clin Endocrinol Metab. 2017;102(2):416-24. doi: 10.1210/jc.2016-2653.

13. Bareti M. Case report of chloroquine therapy and hypoglycaemia in type 1 diabetes: ¿What should we have in mind during the COVID-19 pandemic? Diabetes Metab Syndr. 2020;14(4):355-6. doi: 10.1016/j.dsx.2020.04.014. 